\title{
Electrophysiologic behaviour of a left sided accessory pathway with decremental (Mahaim-like) properties
}

\section{Marco Galeazzi, Maurizio Russo, Sabina Ficili, Carlo Lavalle, Claudio Pandozi}

Dipartimento Cardiovascolare, Ospedale San Filippo Neri, Rome, Italy

Email: m.galeazzi@lycos.com

Received 13 September 2011; revised 10 November 2011; accepted 17 January 2012

\section{ABSTRACT}

The observation of a left sided accessory pathway with decremental properties is rare. We describe the behaviour of one of these pathways which was characterized by the presence of retrograde conduction (not previously reported) and thus inducibility of atrio-ventricular orthodromic reentrant tachycardia.

Keywords: Accessory Pathway; Decremental Properties

\section{INTRODUCTION}

A 52 year-old male was admitted to our cardiology de- partment complaining recurrent episodes of palpitation. The ECG pattern showed sinus rhythm with PR interval $80 \mathrm{msec}$ and signs of minimal ventricular pre-excitation. The electrophysiologic study showed a left lateral eccentric and decremental retrograde conduction. During programmed right atrial stimulation, antegrade long conduction times were detected, along with a progressive leghtening of the A-H interval, a contemporary shortening of the $\mathrm{H}-\mathrm{V}$ interval and a progressive pre-excitation of QRS complexes with right bundle branch block (RBBB) morphology (Figures 1(a)-(b)). The earliest ventricular activation was observed at the level of the distal dipoles of the coronary sinus diagnostic catheter,

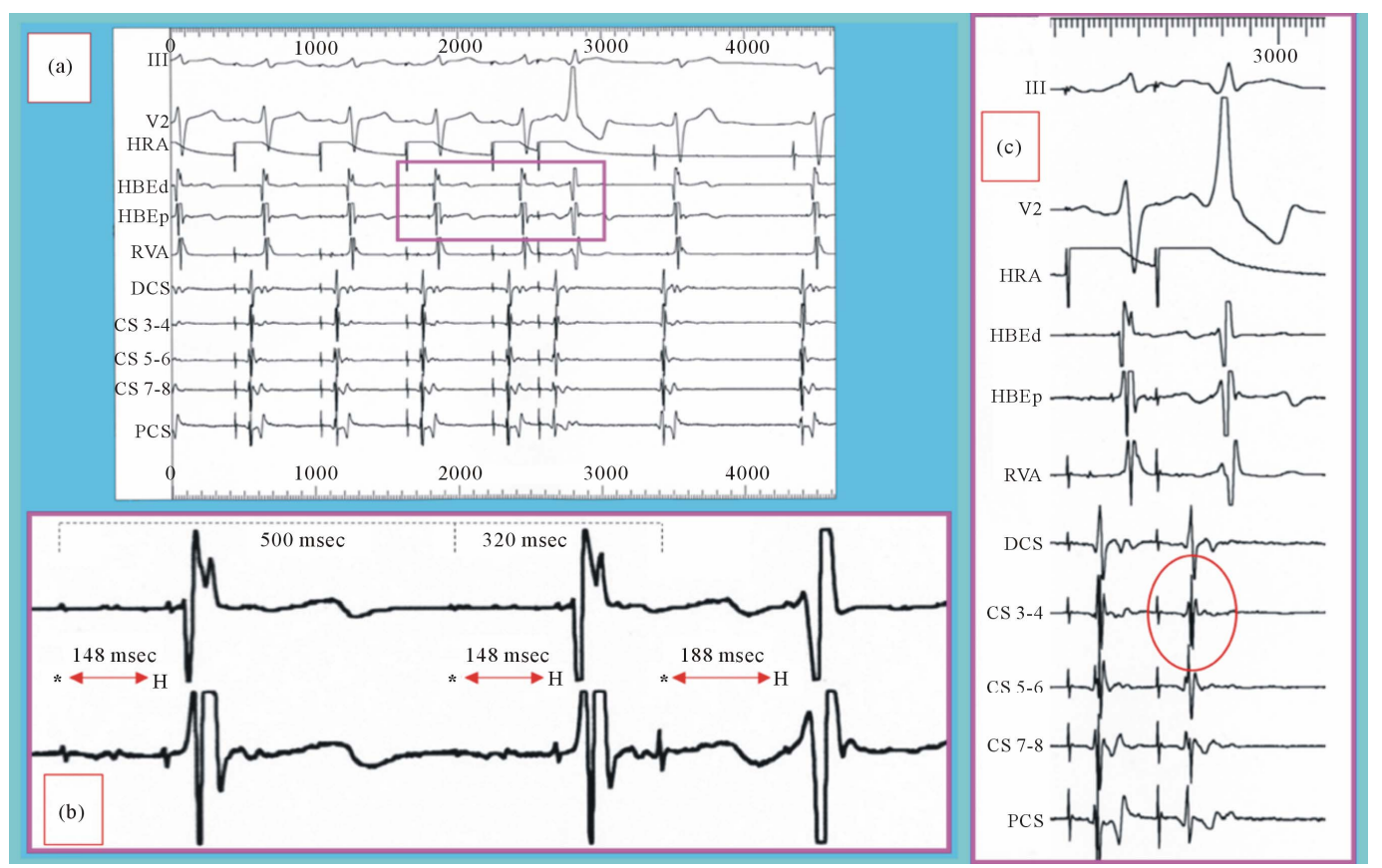

Figure 1. Electrophysiologic study; programmed right atrial stimulation $\left(\mathrm{S}_{0}-\mathrm{S}_{0} 500 \mathrm{msec} ; \mathrm{S}_{0}-\mathrm{S}_{1} 320 \mathrm{msec}\right)$. Leghtening of the spike $(*)-\mathrm{H}$ interval, contemporary shortening of the $\mathrm{H}-\mathrm{V}$ interval ( $\mathrm{H}$ wave moving inside the slow initial component of $\mathrm{V}$ wave), and progressive pre-excitation of QRS complexes with a right bundle branch block (RBBB) pattern are visible (1(a) and 1(b) [details]). The earliest ventricular activation (details in 1(c)) occours at the level of CS 3 - 4 (one of the distal dipoles of the coronary sinus diagnostic catheter, red circle), thus suggesting the presence of a left lateral accessory pathway with decremental properties. HRA = High Right Atrium; HBEd and HBEp = His Bundle catheter Electrodes distal and proximal, respectively; RVA = Right Ventricular Apex; DCS = Distal Coronary Sinus; PCS = Proximal Coronary Sinus; * = spike. 
thus suggesting the hypothesis of a left lateral accessory pathway with decremental properties (Figure 1(c)).

During these manouvers, two different arrhythmias were induced. The former was an antidromic atrioventricular reentrant tachycardia with RBBB morphology (cycle lenght $248 \mathrm{msec}$ ); the site of the earliest ventricular activation was confirmed to be at the level of the left lateral wall (Figure 2). The latter was an orthodromic atrio-ventricular reentrant tachycardia with normal QRS morphology and late progression to an aberrant, RBBB-like ventricular conduction (cycle lenght $282 \mathrm{msec}$ ); also the earliest site of the atrial backactivation was detected at the level of the left lateral wall (Figure 3).

The mitral ring electrical mapping in sinus rhythm allowed the earliest ventricular activation to be recorded at the level of the left lateral wall. The accessory pathway was successfully ablated.

\section{DISCUSSION}

In 1938, Mahaim firstly described the existence of cardiac nodo-ventricular conduction fibres [1]. The behavi- our of these fibres (which are now actually known to be atrio-fascicular or atrio-ventricular) is characterized by antegrade decremental conduction, minimal or no preexcitation during sinus rhythm, absence of retrograde conduction over the accessory pathway, partecipation of the atrium in the antidromic reentrant tachicardia and responsiveness to adenosine. Most of these fibres have been described in the right atrium, although several works do exist concerning the observation of Mahaimlike properties in some left sided accessory pathways [2-7]. Indeed, the electrophysiologic properties of the pathway we describe are not fully compatible with the Mahaim fibres behaviour. The original finding is that a retrograde conduction along the pathway was present, as confirmed by both the basal ventricular stimulation and the induction of an orthodromic reentrant atrio-ventricular tachycardia. Such observation is quite rare and has been previously reported only in a right sided accessory pathway [2].

\section{CONCLUSION}

Although quite rare, findings of retrograde conduction

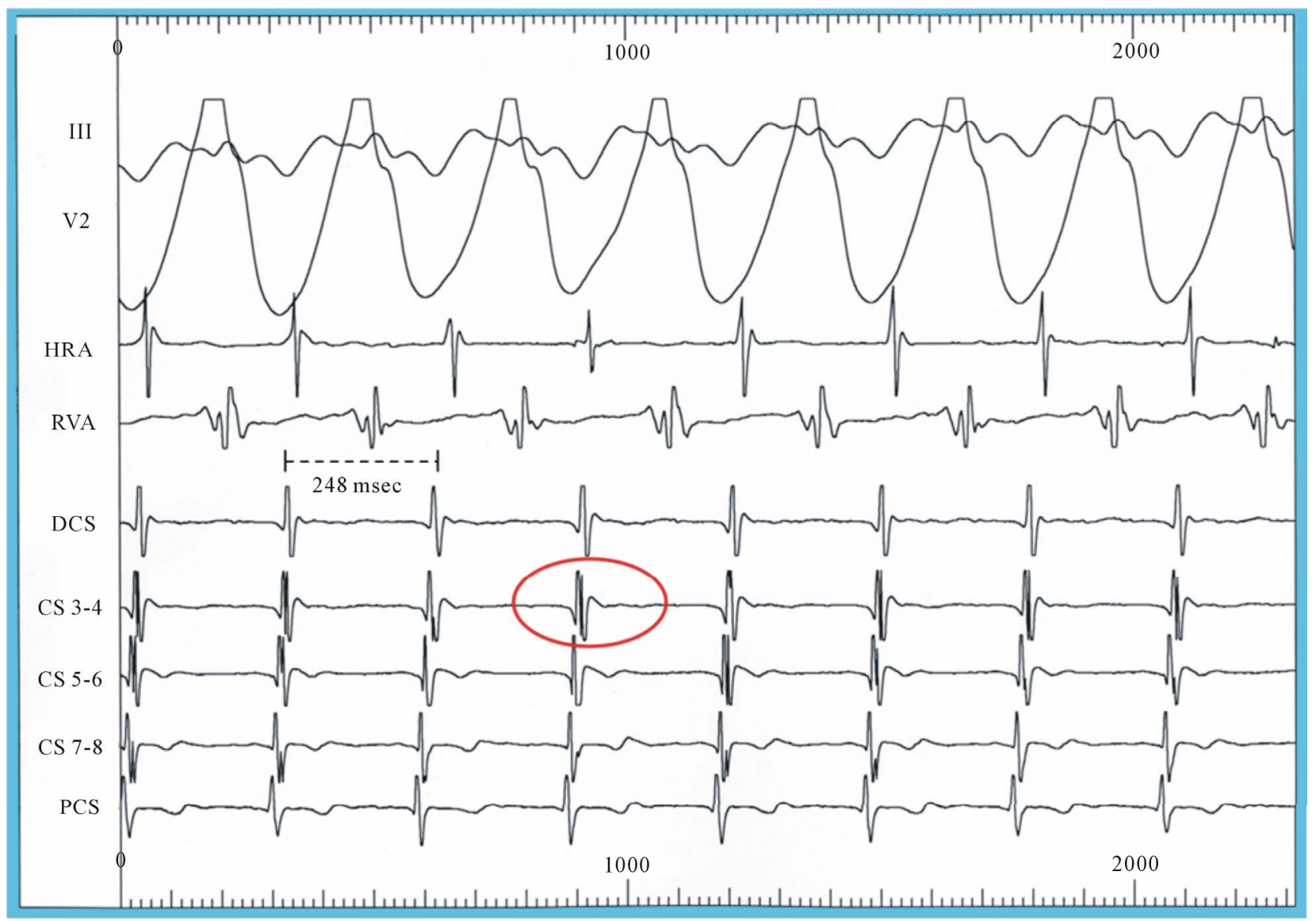

Figure 2. Antidromic atrio-ventricular reentrant tachycardia with RBBB morphology (cycle lenght 248 msec); the site of the earliest ventricular activation is detectable at the level of the left lateral wall (red circle). HRA = High Right Atrium; RVA = Right Ventricular Apex; DCS = Distal Coronary Sinus; PCS = Proximal Coronary Sinus. 


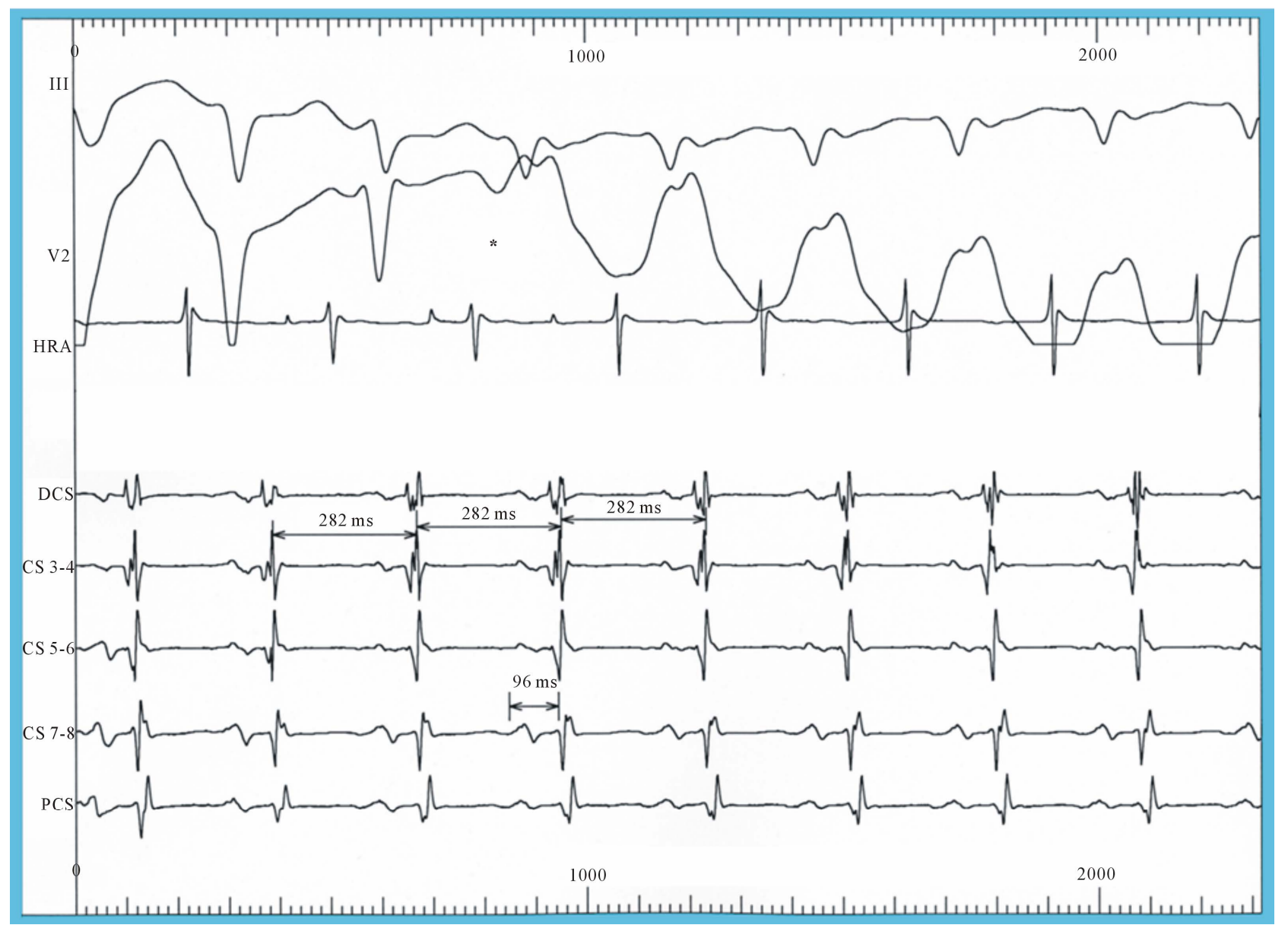

Figure 3. Orthodromic atrio-ventricular reentrant tachycardia with normal QRS morphology and late progression to an aberrant, RBBB-like ventricular conduction (cycle lenght $282 \mathrm{msec}$, red star); the earliest site of the atrial back-activation is detectable at the level of the left lateral wall (CS 3 - 4). HRA = High Right Atrium; DCS = Distal Coronary Sinus; PCS = Proximal Coronary Sinus.

properties in left sided accessory pathways with decremental properties are possible and must be taken into account in the differential diagnosis process concerning orthodromic atrio-ventricular reentrant tachycardias.

\section{REFERENCES}

[1] Mahaim, I. and Benatt, A. (1938) Nouvelles recherches sur les connexions sperieures de la branche gauche du faisceau de His-Tawara avec cloison inter ventriculaire. Cardiologia, 1, 61-120. doi:10.1159/000164567

[2] Kalbfleisch, S., Bowman, K. and Augostini, R. (2008) A single Mahaim fiber causing both antidromic and orthodromic reciprocating tachycardia. Journal of Cardiovascular Electrophysiology, 19, 740-742. doi:10.1111/j.1540-8167.2007.01052.x

[3] Centurion, O.A., Fukatani, M., Shimizu, A., Konoe, A., Isomoto, S., Tanigawa, M., Kaibara, M. and Yano, K. (1993) Anterograde and retrograde decremental conduction over left-sided accessory atrioventricular pathways in the Wolff-Parkinson-White syndrome. American Heart Journal, 125, 1038-1047.

\section{doi:10.1016/0002-8703(93)90112-M}

[4] Osman, F., Stafford, P.J. and Ng, G.A. (2009) Looks like VT but isn't-Successful ablation of a left free wall accessory pathway with mahaim-like properties. Indian Pacing and Electrophysiology Journal, 9, 112-118.

[5] Hluchy, J., Schickel, S., Jörger, U., Jurkovicova, O. and Sabin, G.V. (2000) Electrophysiologic characteristics and radiofrequency ablation of concealed nodofascicular and left anterograde atriofascicular pathways. Journal of Cardiovascular Electrophysiology, 11, 211-217. doi:10.1111/j.1540-8167.2000.tb00323.x

[6] Johnson, C.T., Brooks, C., Jaramillo, J., Mickelsen, S. and Kusumoto, F.M. (1997) A left free-wall, decrementally conducting, atrioventricular (Mahaim) fiber: Diagnosis at electrophysiological study and radiofrequency catheter ablation guided by direct recording of a Mahaim potential. Pacing Clinical Electrophysiology, 20, 2486-2488. doi:10.1111/j.1540-8159.1997.tb06089.x

[7] Yamabe, H., Okumura, K., Minoda, K. and Yasue, H. (1991) Nodoventricular Mahaim fiber connecting to the left ventricle. American Heart Journal, 122, 232-234. doi:10.1016/0002-8703(91)90784-F 